\title{
A VLC-Based Location System for Mobile Application
}

\author{
Priti Konda ${ }^{1}$, Dr. D. K. Shedge ${ }^{2}$ \\ Department of Electronics and Telecommunication, AISSMS Institute of Information Technology , Pune, India ${ }^{1,2}$
}

\begin{abstract}
Visible light communication is the new technology with more benefits over an existing radio frequency communication system such as high security, high energy, efficiency and long life. The primary aim of LED is illumination which is important requirement of VLC system. White light can be used in both indoor and outdoor applications for illumination as well as data transmission because colours of objects as seen closely resemble under both the white light and natural light. The proposed VLC system enables indoor applications by allowing illumination as well as data transmission both of which not affected on each other. The transmitter comprises On-Off keying (OOK) modulation and LED to send data by flashing the light at a flicker-free frequency and speed undetectable to the human eye. In order to avoid flickering in lighting, the designated system should work above $200 \mathrm{~Hz}$. The receiver comprises photodiode and its amplification system followed by Bluetooth module to ensure its connectivity to a smart phone.PWM signals are modulated (Single carrier modulation technique) by the user data and the led driver drive the LED according to the modulated PWM signal.VLC is the peer to peer communication. Therefore, it is enables for several other applications such as home, office, airplane etc.
\end{abstract}

Index Terms: Visible light communication, White light emitting diode, Modulation techniques, Led driver.

\section{INTRODUCTION}

Low energy efficiency is playing vital role in lighting. Recent lighting industries are concentrated on solid state lighting through the light emitting diode. Current fluorescent and incandescent lamps are replaced by LEDs because of its good durability, lower cost, smaller size, more efficient, luminance, cooler operation, and more eco-friendly [1]. Due to these benefits, LEDs adoption will increase in future for illumination purpose. One of the additional advantages of LEDs is that they are switches at very fast rate for the different light intensity [2]. Human eye cannot perceiver faster switching rate such functionality is used for wireless communication where in formation encoded in intensity of light [3]. On other hand photodiodes can receive encoded light in the form of modulated signal for decode data this means that led can provide illumination as well as communication [4]. VLC is green communication which utilizes visible light having a spectral wavelength between 390 to $780 \mathrm{~nm}$ [5]. The visible light spectrum is completely untapped for communication because it is license free bandwidth with hundreds of terahertz. Since visible light is not harmful to humans, animals, and the environment. It has cable of achieving higher data rate in standard of IEEE 802.11.7 [6]. The main dissimilarity between RF and VLC is that in RF information can be encoded in amplitude or phase of the signal of light but in case of VLC, amplitude and phase modulation are not used for data encoding [7]. These characters indicate that, modulation techniques such as phase and amplitude cannot be applied for VLC. This means that the varying intensity of the light wave has to be used for data encoding. In VLC, perceived light by human can be characterized by flicker mitigation and dimming [8]. This is important in VLC because dimming depending on the application which chooses by user, but data modulation should not be affect on the dimming. To provide higher data rate as well as to avoid fluctuations in brightness of the light, flickering should not be sense by human eye. This indicates that in flicker mitigation, change in intensity of light should be faster than $200 \mathrm{~Hz}$.

\section{Characteristic of VLC:-}

Firstly, visible light rays cannot go through walls and objects due to its high frequency. This characteristic allows increasing capacity of communication security. Second, VLC reuse existing lighting infrastructure for communication purpose which indicates that system can deployed at a lower cost with less effort.

\section{A. Light Emitted Diode}

To choose an LED with appropriate specifications is very important factor because it offers both communication and illumination. It is necessary to calculate parameter of LED such as Photometric parameters and radiometric parameter.

Photometric parameter defines the characteristics of light and radiometric parameter measure radiant electromagnet 1) Phosphor coating with Blue LED: In this concept, blue LED used to generate white light with coating of yellow phosphor. When the blue light travels through coating of yellow phosphor, produces a white light with suitable 
combination of blue light and yellow coating. By modifying the thickness of the yellow phosphor coating layer, the variations in white light are produced (color temperatures).

2) RGB Combination: The proper mixing of red, blue and green light can be used to produce white light. In this concept, three separate LEDs are used for three colors respectively, such method define that the cost of LED luminaries is higher as compared to using the Blue LED with coating of phosphor layer. The major constraint of the white LED is its restricted bandwidth. The first method with phosphor coating and blue LED is more commonly used for designing white LED because of ease for implementation and lower cost. However, in terms of speed, communication of LED depends on the phosphor coating because it restricts the speed at which LED can toggle to a few MHz's.

TABLE I Parameters of LED

\begin{tabular}{|l|l|lr|}
\hline Sr. No. & Photometric units & Radiometric units \\
\hline 1 & $\begin{array}{l}\text { Luminous flux [F] } \\
\text { Lumens[lm] }\end{array}$ & $\begin{array}{l}\text { Radiant power } \\
\text { Watts[W] }\end{array}$ \\
\hline 2 & $\begin{array}{l}\text { Luminous intensity [Iv] } \\
\text { Candelas[cd }=1 \mathrm{P} / \mathrm{ster}]\end{array}$ & Radiant intensity[J] \\
Watts per steradian [W/ster] \\
\hline 3 & $\begin{array}{l}\text { Illuminance [E] } \\
\text { Lux [lx }=1 \mathrm{~m} / \mathrm{m} 2]\end{array}$ & $\begin{array}{l}\text { Irradiance } \\
\text { Watts per square metre [W/m2] }\end{array}$ \\
\hline
\end{tabular}

B. Modulation technique

There are 2 types of modulation techniques such as single carrier and multicarrier. Single carrier modulation technique consist On-Off Keying (OOK) and Pulse Modulation method. On other hand multicarrier modulation techniques include Orthogonal Frequency Division. The hardware implementation of OFDM is very complex, expensive, time consuming and west full for low data rate application. So single carrier modulation is commonly applied to VLC

1) Single carrier modulation

On-Off Keying (OOK): In OOK modulation, ON state of LED represented by data bit"1"and OFF state by " 0 ". In the OFF state, intensity of light is reduced because LED is not OFF completely. OOK is simple and easy for implementation and it widely used in application of wire line communication.

\section{Pulse Modulation:}

Pulse width modulation: In PWM, based on dimming level the width of the pulses is adjusted. The signal which is encoded by data that is modulated signal transmitted during pulses. Based on the dimming requirement, the data rate of modulated signal is adjusted. In which dimming is obtained without changing the intensity level of pulse.

Pulse position modulation: In this technique, the symbol duration is divided into equal duration of $\mathrm{t}$ slots and a pulse is transmitted in one of the $t$ slots. The position of the pulse identifies the transmitted symbol. It widely used in infrared communication but have limitation of lower spectral efficiency and data rate of PPM. The further implementation of ppm is Overlapping PPM (OPPM). During symbol duration more than one pulse to be allows for transmission. It can provide high data rate but not achieve higher spectral efficiency. In Another case, multiple pulses to be transmitted during the symbol duration are called as Multipulse PPM (MPPM) which can provide a higher spectral efficiency compared to OPPM.

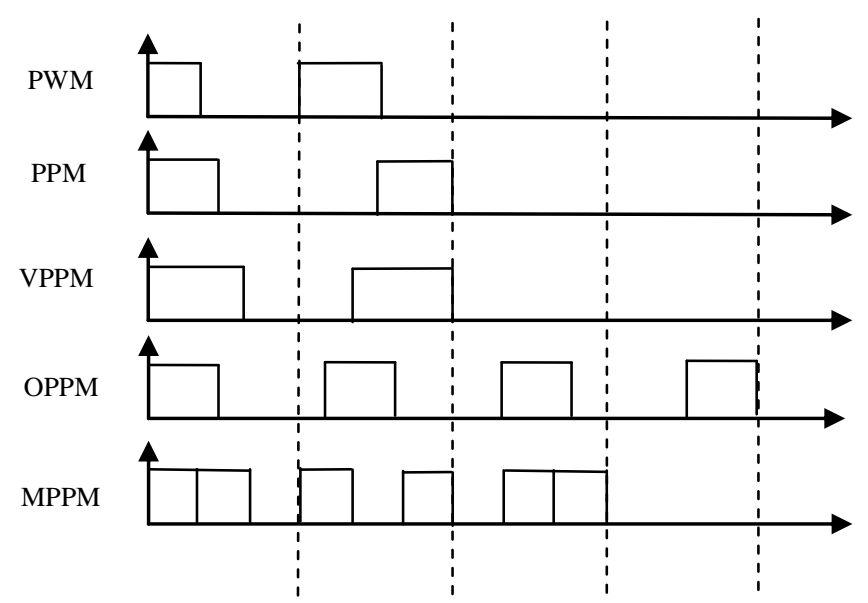

Fig. 1 Magnetization Waveform of modulation technique 
Variable PPM (VPPM) was proposed by IEEE 802.15.7 standard which combines PPM and PWM scheme which shown in figure1. In VPPM, different position of pulses are choose to encode the bits as in PPM, however, the width of the pulse can also be modified as needed. It allows different dimming levels by altering the pulse width is simple and robustness.

\section{2) Multi carrier modulation}

Orthogonal Frequency Division Multiplexing (OFDM): Single-carrier modulation scheme suffer from high intersymbol interference because of non-linear frequency response of visible light communication channels. Therefore OFDM can be used to reduce the inter-symbol interference in VLC and which has been widely adopted in the RF communication. In OFDM, the channel is divided into multiple orthogonal subcarriers and such carrier modulated by data which is sent in parallel sub streams. Non-linearity of LED is biggest challenge in OFDM based VLC system which define relationship between the current and the emitted light of the LED is non linear. The solution to this challenge is that LED operated in small range where the driving current and optical power is quasi-linear.

Color Shift Keying (CSK): White light can be generated using three separate LEDs. These three LEDs are Red, Green, and Blue respectively. The combination of these three RGB LEDs produces one combined source which referred as TriLED (TLED). In CSK, intensity of the three colors in the TLED source can be used to modulate the signal. For visible light communication, IEEE 802.15.7 standard proposed CSK modulation. It widely used to overcome the lower data rate and limited dimming support issues. In CSK, dimming support is simply amplitude dimming where the driving current of the LEDs is varied to change the brightness of resultant white light.

Advantage of CSK is that wavelength multiplexing method through which the multiple users can be access.

\section{VLC SYSTEM OVERVIEW}

The primary objective of LEDs is to provide illumination which should not affect during communication. When led is used in illumination mode, the driver circuit controls the current flowing through LED and when it is in communication mode, the data can be modulate through the use oflight using the driver circuit. For example, in a simple on-off keying modulation, 0 bit data represent OFF mode of LED and 1 bit data represent ON mode of led. This means ON and OFF modes are represents two intensity levels of light. The design system of VLC consist power supply, analog circuitry, microcontroller. Analog circuitry incorporate led, led driver, photodiode, amplifier circuit etc shown in figure 2.

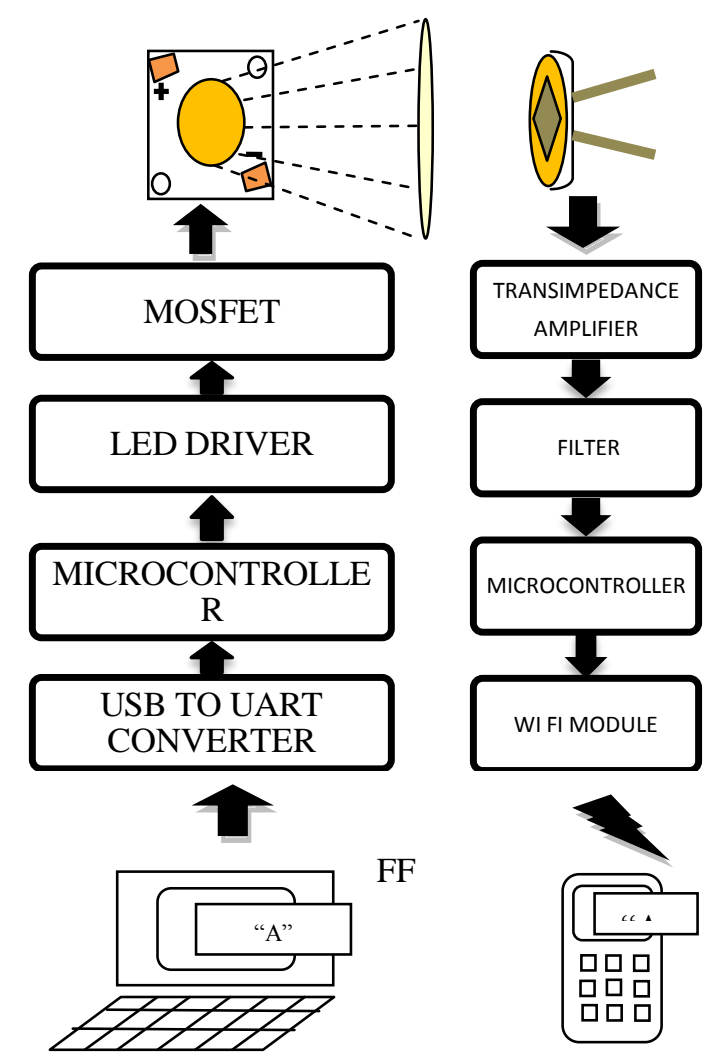

Fig. 2 Block Diagram of Transmitter and Receiver Circuit 
At the transmitter side data stream from PC which sent to the microcontroller. The communication between PC and microcontroller is using USB to UART converter [9]. The transmitter microcontroller modulated data stream using OOK scheme for logic 1 and logic 0 and send to the driver to drive LEDs according to data. At receiver side, the photo detector is a semiconductor device whose switching frequency should be less than 100ns and wavelength in the range of $400 \mathrm{~nm}$ to1100nm. It converts the received light into current which is in analog form. Resister divider and Transimpedance amplifier circuit are used to convert analog signal output from photodiode to digital form and amplify the signal respectively. Such amplify signal will be given to Receiver microcontroller which decode the digital signal into UART frame and transfer to mobile via Bluetooth. In VLC structure, LED is employed as the antenna of transmitter with good light modulation performance. PD is a semiconductor device for converting light into electric current in high speed. A PD with response time in nanosecond even picoseconds magnitude can provide an available bandwidth for signal modulation and data transmission when it is used in a VLC system.

\section{IMPLEMENTATIONS}

\section{A. Hardware}

The hardware part in the proposed system design is shown in figure 3.The transmitter comprises PIC18F4S20, AC TO DC converter, MOSFET, DC to DC converter, LED, LCD, RS232 etc. To power the microcontroller and LED voltage conversion is required. First, an ACDC converter used to convert 230AC into 12 VDC and again convert this 12VDC into 5DC to power the PIC18F4S20. RS232 is communication cable which generally used to transfer and receive the serial data between PC and microcontroller. It is interfacing with the microcontroller using MAX232 IC, which act as a buffer driver for controller. Output of Microcontroller is used to drive LED.

The receiver comprises PIC18F4S20, Photodiode, LPF, Amplifier AC TO DC converter, LCD,DC to DC converter, Bluetooth and Mobile etc. photodiode is used covert light signal into electrical signal. Output of photodiode is electrical current which convert into a voltage. It fed LPC to overcome distortion and amplify the signal by using amplifier. Such amplified signal fed as an input to PIC18F4S20, which decode the data and send to the mobile via Bluetooth.
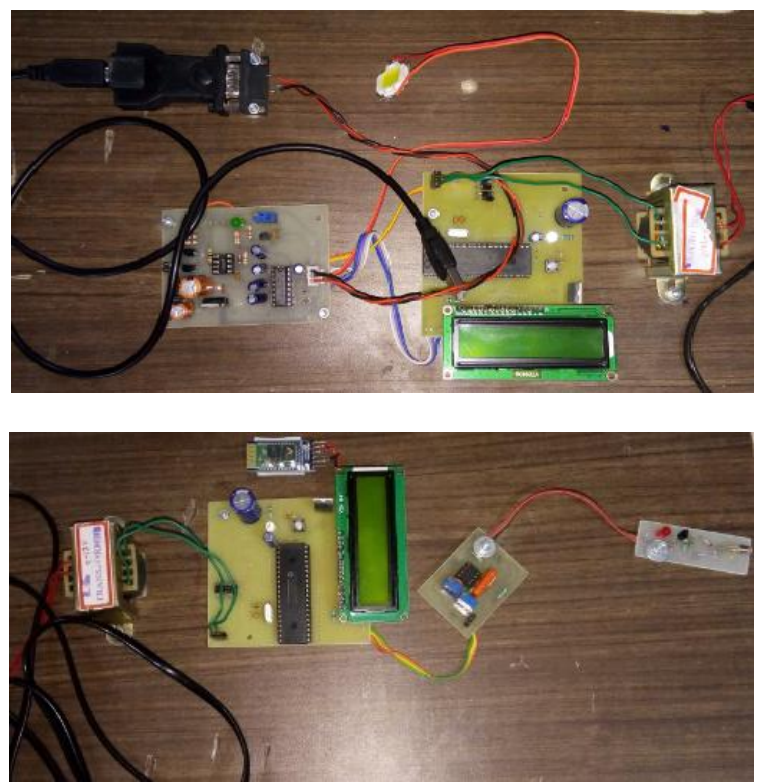

Fig. 3 hardware of Transmitter and Receiver Circuit

\section{B. Software}

The software part consist Hyper Terminal and MikroC Pro for PC and microcontroller respectively. The software designated for PC that used for user interface so that the user can type the data in the HyperTerminal in the form of text paragraph up to 1000 characters. It also provides data frame to controller but does not accumulate on the microcontroller. The software designed for microcontroller consist data buffer, data conversion from ASCII into binary bit stream, data framing for synchronization, and data modulation. The flowchart on transmitter software design is shown in figure 4.

On the side data packet is received on receiver side which has been detected. The detected data packed is demodulated and converted into ASCII characters which display on the LCD as well as send to the Bluetooth device. 

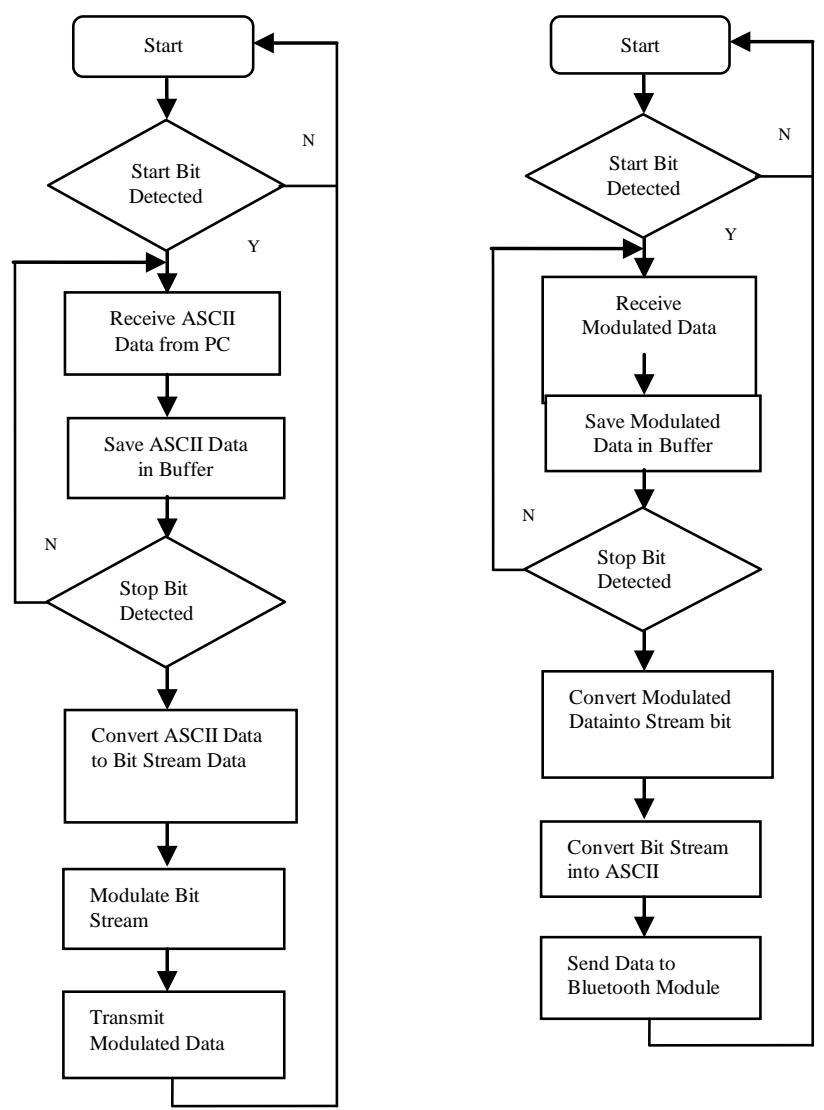

Fig. 4 flowchart of software on transmitter and receiver

\section{CONCLUSION}

Visible light communication technology provides energy efficiency and high speed data with security/privacy. Different modulation techniques are discussed to provide dimming and minimize flickering effect with high spectral efficiency. The method of transmitting information with illumination using visible light communication has been shown successfully.The transmission of information at speeds up to $1200 \mathrm{bps}$ with a very low BER using our suitable components and simple system. It reaches $70 \%$ level of accuracy within $40 \mathrm{~cm}$ distance.

\section{ACKNOWLEDGMENT}

The author would like to thanks Dr. D. K. Shedge for his support and guide to develop different idea which are valuable to complete this project work, also for his inspiration.

\section{REFERENCES}

[1] T. Komine an M. nakagawa," Fundamental Analysis for Visible Light Communication System Using LED Lights," IEEE transactions Consumer Electronics, vol. 50, no. 1, pp. 100-107,2004Electronics, vol. 50, no. 1, pp. 100-107, 2004.

[2] H. Sugiyama, S. Haruyama,"Brightness Control Methods for Illumination and Visible Light Communication Systems," in Third International Conference on Wireless and Mobile Communications 2007 (ICWMC'07). IEEE,2007 pp.78-78.

[3] Liu Xian, Yang Aiying, "Dimming Controlling and Data Transmission for an Indoor Visible Light Communication System," China Communication, March 2015.

[4] Angga Pradana, and Nur Ahmadi, "Implementation of Visible Light Communication System Using Pulse Width"The 5th International Conference on Electrical Engineering and Informatics, August 10-11, 2015, Bali,

[5] Indonesia.Kevin Warmerdam, Ashish pandharipande, "Visble Light Communications for Sensing and Lighting Control," IEEE sSensor Journal, vol.16, no.17, September 1, 2016.

[6] M. V. Bhalerao, S. S. sonavane , "A Suvey of Wirless Communication Using Visible Light," Internation Journal of Advance in Engineering \& Techonolgy, vol-5, issue-2, pp:199-197, January 2013.

[7] John Gancarz, Hany Elgala, "Impact of Lighting Requirements on VLC Systems," IEEE Communication Magazine, December 2013.

[8] Panarat Cherntanomwong, Wisarut Chantharasena, "Indoor Localization System Using Visible Light Communication,"7th International Conference on Information Technology and Electrical Engineering (ICITEE), Chiang Mai,Thailand, 2015.

[9] Deng Jian-zhi, Yao Meng, “A Real-Time VLC to UART Protocol Conversion System,” Optoelectronics Letters vol.12, no.4, 1 July 2016. 